\title{
Migration of Perilesional Microglia after Focal Brain Injury and Modulation by CC Chemokine Receptor 5: An In Situ Time-Lapse Confocal Imaging Study
}

\author{
W. Shawn Carbonell, ${ }^{1,2}$ Shin-Ichi Murase, ${ }^{3}$ Alan F. Horwitz, ${ }^{2,3}$ and James W. Mandell ${ }^{2,4}$ \\ ${ }^{1}$ Medical Scientist Training Program, ${ }^{2}$ Neuroscience Graduate Program, ${ }^{3}$ Department of Cell and Molecular Biology, and ${ }^{4}$ Department of Pathology, \\ Division of Neuropathology, University of Virginia Health System, Charlottesville, Virginia 22908
}

\begin{abstract}
Microglia rapidly become reactive in response to diverse stimuli and are thought to be prominent participants in the pathophysiology of both acute injury and chronic neurological diseases. However, mature microglial reactions to a focal lesion have not been characterized dynamically in adult vertebrate tissue. Here, we present a detailed analysis of long-distance perilesional microglial migration using time-lapse confocal microscopy in acutely isolated living slices from adult brain-injured mice. Extensive migration of perilesional microglia was apparent by $24 \mathrm{~h}$ after injury and peaked at $3 \mathrm{~d}$. Average instantaneous migration speeds of $\sim 5 \mu \mathrm{m} / \mathrm{min}$ and peak speeds $>10 \mu \mathrm{m} / \mathrm{min}$ were observed. Collective, directed migration toward the lesion edge was not observed as might be expected in the presence of chemoattractive gradients. Rather, migration was autonomous and could be modeled as a random walk. Pharmacological blockade of the cysteine-cysteine chemokine receptor 5 reduced migration velocity and the number of perilesional migratory microglia without affecting directional persistence, suggesting a novel role for chemokines in modulation of discrete migratory parameters. Finally, activated microglia in the denervated hippocampal stratum oriens did not migrate extensively, whereas human immunodeficiency virus-1 tat-activated microglia migrated nearly twice as fast as those at the stab lesion, indicating a nonuniform microglial response to different stimuli. Understanding the characteristics and specific molecular mechanisms underlying microglial migration after neural injury could reveal novel targets for therapeutic strategies for modulating neuroinflammation in human diseases.
\end{abstract}

Key words: brain injury; chemokines; microglia; migration; neuroinflammation; reactive gliosis

\section{Introduction}

Microglia are thought to represent resident brain tissue macrophage precursors, which rapidly respond to diverse insults in a stereotyped manner (Kreutzberg, 1996; Becher et al., 2000). Under normal conditions, microglia exist as nonmigratory ramified cells but rapidly transform into a motile amoeboid form within hours of activation (Stence et al., 2001; Nimmerjahn et al., 2005). These activated cells are presumed to migrate toward tissue damage and transform into phagocytes. The importance of microglial function in acute neurological insults such as traumatic brain injury (TBI) is well documented (Kreutzberg, 1996; Becher et al., 2000). This is underscored by recent microarray studies, which

\footnotetext{
Received Dec. 19, 2004; revised June 9, 2005; accepted June 9, 2005.

This work was supported by National Institutes of Health (NIH) Grants NS-047378 (J.W.M.), GM-232442 (A.F.H.), and GM-064346 (A.F.H.). W.S.C. was supported by the University of Virginia Medical Scientist Training Program, NIH Training Grant Award 5 T32 GM07267-24, a Raven Society Fellowship, a National Neurotrauma Society Student Award, and a University of Virginia Graduate School of Arts and Sciences Paper Award. TAK-779 and recombinant HIV-1 tat protein (Dr. John Brady) were obtained through the NIH AIDS Research and Reference Reagent Program, Division of AIDS, National Institute of Allergy and Infectious Diseases, NIH (http://www.aidsreagent.org). We thank Rooshin Dalal, Claire Brown, and Akira Sakakibara for helpful discussion and expert assistance with confocal microscopy. A low-power, coronal photomicrograph of the C57BL/6 mouse brain was used, courtesy of mbl.org.

Correspondence should be addressed to Dr. James W. Mandell, Department of Pathology, Division of Neuropathology, University of Virginia Health System, MR-5, Room 3220, P.0. Box 800904, Charlottesville, VA 22908. E-mail: jwm2m@virginia.edu.

DOI:10.1523/JNEUROSCI.5171-04.2005

Copyright $\odot 2005$ Society for Neuroscience $\quad$ 0270-6474/05/257040-08\$15.00/0
}

show the largest changes in gene expression in several different animal models of TBI to be inflammation related (Kobori et al., 2002; Natale et al., 2003). Prominent roles for microglia and neuroinflammation have also been hypothesized in chronic neurological diseases in humans and animal models such as Alzheimer's disease, human immunodeficiency virus-1 (HIV-1) encephalopathy, prion diseases, and multiple sclerosis (Benveniste, 1997; Becher et al., 2000; Nelson et al., 2002; Streit et al., 2004; Lee et al., 2005).

Recent attempts to study microglial dynamics in mammalian tissue have been informative but restricted to acute reactions induced by tissue slicing (Nimmerjahn et al., 2005). These studies have demonstrated the transformation of ramified microglia to more motile, amoeboid forms (Stence et al., 2001) but only limited analysis of microglial migration (Stence et al., 2001; Grossmann et al., 2002; Petersen and Dailey, 2004). Studies in the leech have provided clues about migratory characteristics and mechanisms of microglia in the invertebrate nerve cord, but the relevance of these findings to mammalian systems has not been verified (McGlade-McCulloh et al., 1989; Peters et al., 1991; Duan et al., 2003).

There exist no dynamic analyses of more mature reactive microglial functions in adult mammalian tissue after focal injury. Thus, the dynamic in situ characteristics and mechanisms of microglial migration remain primarily unknown. Foremost, the 
idea that the microglial response to focal injury is characterized by directed migration toward the lesion followed by transformation into phagocytes has not been verified in vertebrate tissue. In addition, the role of cysteine-cysteine (CC) chemokines in focal lesion is unclear (Ghirnikar et al., 1996; Hausmann et al., 1998). These cytokines are thought to guide blood monocytes to the injured brain but may also play a direct role in promoting directional and collective migration of resident brain immune cells, the microglia, as predicted by studies in vitro (Hausmann et al., 1998; Marella and Chabry, 2004). It is also not known for how long activated cells retain the capacity to migrate after injury or the migration strategies used. Finally, the concept of a uniform microglial response in the face of different types of injury has not been investigated by direct observation.

Here, we address these questions in acutely isolated living slices taken from in vivo brain-injured adult mice at various postlesion intervals. These results define perilesional microglial migratory behavior and regulation in living adult mammalian tissue after focal neural injury.

\section{Materials and Methods}

Animals. Adult male C57BL/6 mice (6-8 weeks of age) were obtained commercially (Harlan, Indianapolis, IN). All animals were cared for in accordance with United States Public Health Service guidelines.

Stab lesion. Forebrain stab lesion (FSL) was performed as described previously (Carbonell and Mandell, 2003).

Intracerebroventricular injections. Recombinant HIV-1 tat protein (2.0 $\mathrm{nm})$ in $100 \mathrm{mg} / \mathrm{ml} \mathrm{BSA}$ and $0.1 \mathrm{~mm}$ DTT in PBS $(n=6)$ were stereotaxically injected into the left lateral ventricle. Tat vehicle alone $(100 \mathrm{mg} / \mathrm{ml}$ BSA, $0.1 \mathrm{~mm}$ DTT in PBS; $n=2)$ or deactivated tat $(n=4)$ were used as volume-matched controls. Deactivated tat was prepared by exposing the $2.0 \mathrm{~nm}$ tat solution to light and air under a culture hood overnight (Fanales-Belasio et al., 2002).

Preparation of live brain slices. Acutely isolated coronal brain slices were collected in chilled physiological saline with a vibratome at 1, 2, 3, 5, or $7 \mathrm{~d}$ after FSL ( $n=16,2,3,2$, and 2, respectively) as described previously (Murase and Horwitz, 2002) with minor modifications. We used $300 \mu \mathrm{m}$ slices in these experiments. Migration media consisted of cell culture media-1 (Hyclone, Logan, UT) with 20\% heat-inactivated normal horse serum. Vital labeling of microglia was performed with $5 \mu \mathrm{g} / \mathrm{ml}$ Alexa 488 or $568 \mathrm{IB}_{4}$ (Molecular Probes, Eugene, OR) in migration media as described previously (Stence et al., 2001). For vital nuclear staining of live slices, 2- $6 \mu \mathrm{M}$ Syto 16 (Molecular Probes) was added to the media for at least $2 \mathrm{~h}$ at $37^{\circ} \mathrm{C}$ followed by a brief rinse. Before recording, slices were covered with $200-500 \mu \mathrm{l}$ of growth factor-reduced Matrigel (BD Biosciences, San Jose, CA) and gelled at $37^{\circ} \mathrm{C}$ for $15 \mathrm{~min}$ to minimize tissue settling effects/motion artifact during recording. Temporal quantitation of microglial migration was derived from measurements from three to four independent experiments per time point from slices taken from at least two different mice. For consistency between time points, quantitated subfields were all centered on the lesion.

Confocal time-lapse videomicrography and analysis. Laser-scanning confocal images were acquired on a Nikon IX-70 inverted microscope with Fluoview 300 software (Fig. 1) (Olympus Optical, Melville, NY). A 20 or $40 \times$ long working distance objective (Olympus Optical) was used in these experiments corresponding to $x-y$ tissue fields measuring $175 \times$ 175 and $350 \times 350 \mu \mathrm{m}$, respectively. The microscope stage and objective were maintained at $37^{\circ} \mathrm{C}$. A $z$-series stack covering $40 \mu \mathrm{m}$ of slice thickness was taken every $1.5 \mathrm{~min}$, creating a three-dimensional time-lapse data set. To create time-lapse movies from the data set, four to six $z$-plane images were collapsed as two-dimensional projections using Image $1.32 \mathrm{j}$ (Wayne Rasband, National Institutes of Health, Bethesda, MD; http:// rsb.info.nih.gov/ij/) and compiled into quick-time movies with Quicktime Pro 6.3 and 7.0.1 (Apple Computers, Cupertino, CA). For quantitative analysis, we identified 47 long-distance (i.e., continuously) migratory cells from four independent 90 min experiments representing tissue from four different mice. A portion of one of these raw movies is

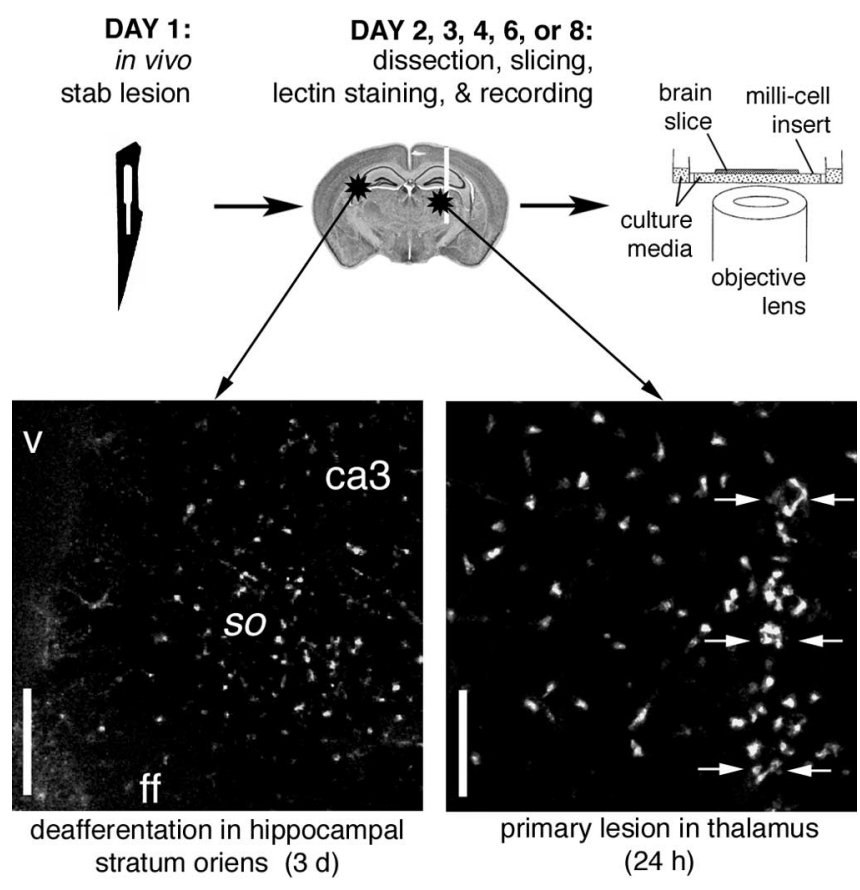

Figure 1. Procedures for confocal time-lapse videomicrography of microglia after focal lesion. Adult mice were subjected to forebrain stab lesion on the first day. One to $7 \mathrm{~d}$ after injury, the brains were rapidly collected, sliced coronally at $300 \mu \mathrm{m}$ with a vibratome, and stained with fluorescently tagged $\mathrm{IB}_{4}$ lectin to visualize microglia/macrophages. Confocal recording was performed for $1-4 \mathrm{~h}$ in the regions denoted by the black stars. Microglia become activated in response to both indirect and direct damage produced by forebrain stab lesion. Bottom right, Activated microglia visualized with $\mathrm{IB}_{4}$ lectin at the stab lesion are widespread in the ipsilateral thalamus (white arrows; stab lesion). Scale bar, $50 \mu \mathrm{m}$. Bottom left, Activated microglia responding to deafferentation can be found in the stratum oriens of the hippocampus in the contralateral hemisphere by $3 \mathrm{~d}$. Scale bar, $100 \mu \mathrm{m}$. ca3, Stratum pyramidale of the hippocampus; ff, ventromedial fimbria/fornix; so, stratum oriens of the hippocampus; v, lateral ventricle.

available for viewing (movie 9, available at www.jneurosci.org as supplemental material). To estimate speed ( $S$ ), the centroid of the migrating cell was traced at $90 \mathrm{~s}$ intervals over a $90 \mathrm{~min}$ period using ImageJ $1.32 \mathrm{j}$ (Ware et al., 1998; Knight et al., 2000; Murase and Horwitz, 2002) to derive the mean squared displacements. Instantaneous speed was derived from absolute displacements per interval (interval, $90 \mathrm{~s}$ ) for each cell. These were corrected to reflect velocities in micrometers per minute. Persistent random walk (PRW) analysis and Chemotactic Index (CI) were performed as described previously (Maheshwari and Lauffenburger, 1998; Ware et al., 1998). All values are reported as the mean \pm SEM. Rose plots, manual plots, and histograms were created in Kaleidagraph 3.0 (Abelbeck Software, Reading, PA). Automated multiple cell-track tracings were performed with the MTrack2 plug-in for ImageJ 1.32j.

Inhibition of CC chemokine receptor 5 and data analysis. Recording was performed in three phases from each slice. After an initial baseline recording experiment, 0 , 4, or $40 \mu \mathrm{M}$ TAK-779, the highly selective, smallmolecule CC chemokine receptor 5 (CCR5) inhibitor (Baba et al., 1999, Marella and Chabry, 2004) in solvent of water, was added to the migration media and incubated for $3 \mathrm{~h}$ at $37^{\circ} \mathrm{C}$. Recording was resumed in the identical field for $60 \mathrm{~min}$ in the continued presence of a drug. For drug washout, the slice was rinsed briefly in two changes of migration media and then cultured for $24 \mathrm{~h}$. For the final recording phase, the migration media were replaced, the original recording field was then identified, and acquisition continued for $\geq 60 \mathrm{~min}$. Experiments were performed in triplicate in tissue slices from five different mice. Percentage of microglial migration represents the average number of migratory cells from three $150 \times 150 \mu \mathrm{m}$ subfields during $1 \mathrm{~h}$ of recording. We operationally defined a migratory cell as one demonstrating a net translocation of at least two cell lengths during the sampling period. Directional protrusive events were scored to determine effects on polarity establishment. One 
point was given for each dominant protrusive event or directional orientation change that occurred $\geq 90^{\circ}$ from the previous direction. PRW analysis was performed as above on continuous migrators from $30 \mathrm{~min}$ migration episodes in each condition. Data analysis was performed with ANOVA, and post hoc pairwise comparisons were made with a $t$ test with the Bonferroni correction factor. For all comparisons, $p<0.05$ was considered statistically significant.

\section{Results}

\section{Focal lesion incites extensive perilesional} microglial migration

To determine the optimal time period for discrete analysis of microglial migratory behavior, we recorded from slices taken 1, 2, 3,5 , and $7 \mathrm{~d}$ after lesion and quantified microglial migration (Fig. $2 \mathrm{~A}$ ). In the perilesional thalamus $1 \mathrm{~d}$ after injury, the majority of $\mathrm{IB}_{4}$-positive microglia were motile and many were highly migratory (Fig. 2B, left) (movies 1 and 9, available at www.jneurosci.org as supplemental material). The numbers of migratory cells increased over subsequent time points peaking at $3 \mathrm{~d}$ (Fig. $2 \mathrm{~A}, \mathrm{~B}$, middle) (movies 2 and 3, available at www.jneurosci.org as supplemental material). However, migration of cells was greatly reduced at 5 and $7 \mathrm{~d}$ after lesion (Fig. $2 \mathrm{~A}$ ) (movies 4 and 5, available at www.jneurosci.org as supplemental material). Thus, widespread migration of activated perilesional microglia occurs by $1 \mathrm{~d}$ after stab lesion with a peak at $3 \mathrm{~d}$.

To verify that the robust microglial responses we observed here were mainly a result of the FSL rather than the brain-slicing procedure, we recorded from the injured and uninjured thalamus of slices $24 \mathrm{~h}$ after FSL to compare the extent of cellular migration. We used Syto-16, a vital nuclear dye, as a label independent of microglial activation in these experiments. As expected, migration of Syto-16-labeled cells was observed in the injured, but not the contralateral, thalamus (Fig. 2C) (movie 6, available at www.jneurosci.org as supplemental material). In addition, when movies are captured in the cortex at midline, it is possible to examine the injured and uninjured cortices simultaneously. In doing so, we verified that microglial activation and migration were limited to the injured hemisphere with both $\mathrm{IB}_{4}$ (movie 7, available at www.jneurosci.org as supplemental material) and Syto 16 (data not shown). Finally, we collected slices from a naive, uninjured mouse and recorded from several thalamic fields from three slices after $24 \mathrm{~h}$ of acute culture. Microglial activation was minimal as revealed by the low- $\mathrm{IB}_{4}$-staining intensity, and only a single cell was found to be migratory (movie 8 , available at www.jneurosci.org as supplemental material). Thus, these results indicate that the migration observed perilesionally after FSL was attributable to a mature microglial response established in vivo and that the slicing procedure itself does not acutely induce significant migration of microglia in the adult mouse brain.

\section{Characterization of perilesional microglial locomotion at $\mathbf{1} \mathbf{d}$}

To characterize perilesional microglial migration in detail, we analyzed time-lapse experiments from mice killed $1 \mathrm{~d}$ after injury (supplemental Table 1, available at www.jneurosci.org as supplemental material). The $3 \mathrm{~d}$ time point was not selected because of the vastly increased numbers of migratory cells, which complicated discrete two-dimensional analysis (e.g., overlapping paths, increased interactions). A portion of a typical experiment in $1 \mathrm{~d}$ tissue may be viewed on-line (movie 9, available at www.jneurosci.org as supplemental material). We found, on average, that $57 \pm 3 \% \mathrm{IB}_{4}$-positive cells in a $40 \times$ perilesional field were migratory. Approximately 30\% were long-distance migrators (see Ma-
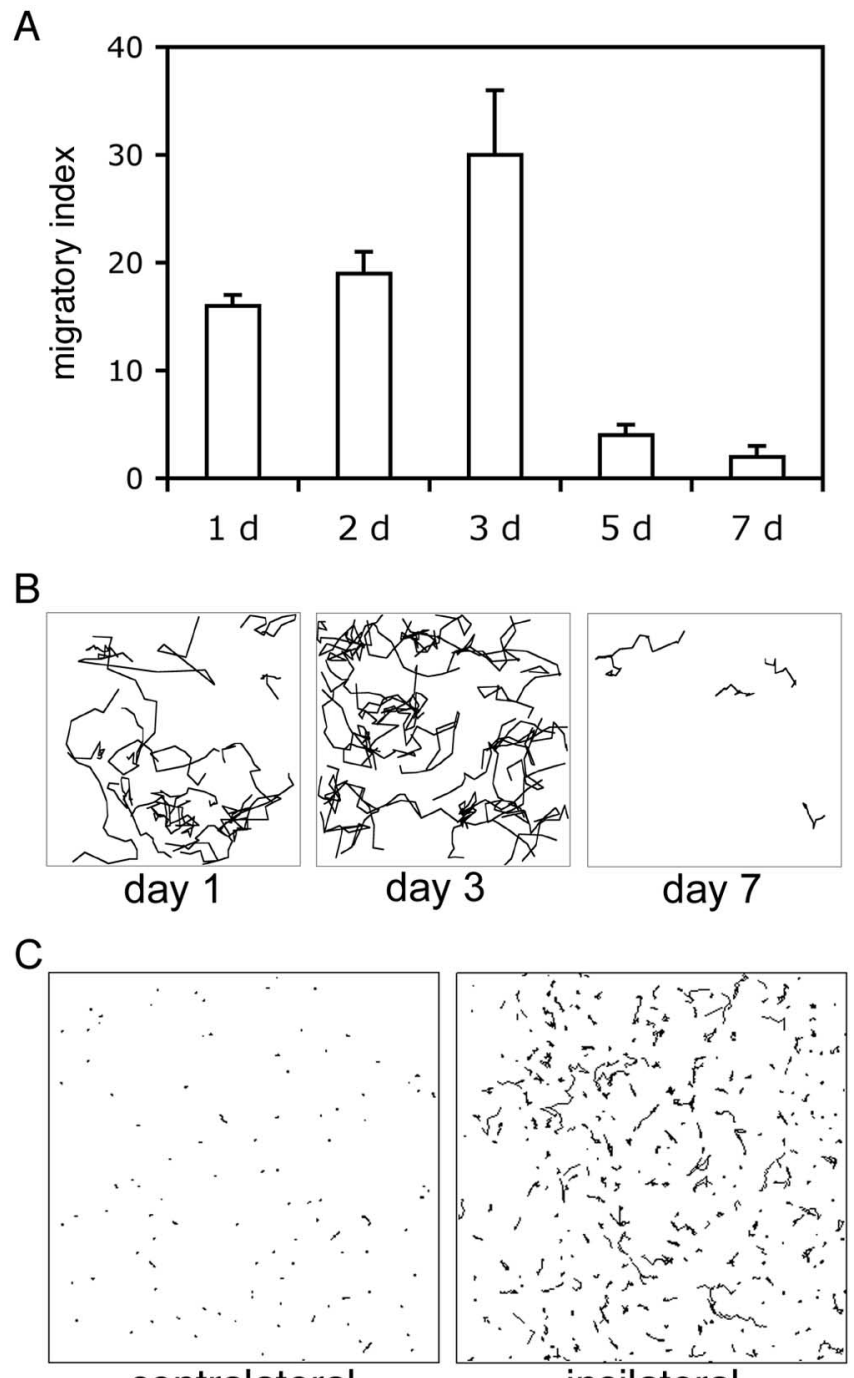

contralateral

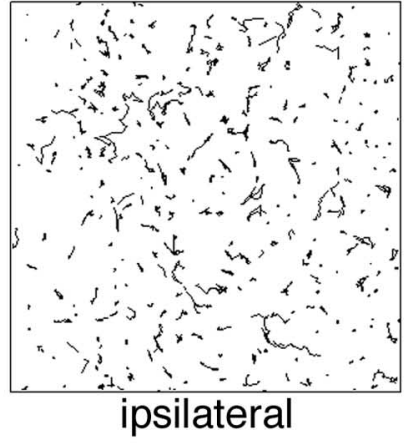

Figure 2. Characterization of perilesional migration. $A$, The peak of cellular migration occurs at day 3 with substantial decline by day 7 . The migratory index represents the mean migratory cell number per $75 \times 75 \mu \mathrm{m}$ subfield. See movies 1-5 (available at www.jneurosci.org as supplemental material). $\boldsymbol{B}$, Representative manual cell path tracings of migratory microglia from quantitated subfields at 1,3 , and $7 \mathrm{~d}$ after lesion. C, Cellular migration is not caused by the brain slicing procedure. Automated cell track tracings were performed from slices vitally stained with Syto16 for nuclei in $300 \times 300 \mu \mathrm{m}$ fields sampled from the uninjured (left) or injured (right) thalamus $24 \mathrm{~h}$ after lesion (fields are from the identical slice; $n=3$ independent experiments). Migratory cells were only found in the injured hemisphere, demonstrating that the slicing procedure itself did not acutely stimulate migration. See movie 6 (available at www. jneurosci.org as supplemental material).

terials and Methods) and are the basis of the quantitative analysis below. This long-distance migration was mostly continuous and meandering (movies 1-5, 9, available at www.jneurosci.org as supplemental material). Migratory cells, which stopped for extended periods, often did so to interact with other cells, vessels, or the primary lesion (movie 9, available at www.jneurosci.org as supplemental material). Morphology of migratory cells varied, but most displayed transient forward protrusions and often longtrailing processes (Fig. 3A) (movie 10, available at www.jneurosci. org as supplemental material). Cells at the edge of the lesion were mostly nonmigratory and amoeboid in morphology and displayed behavior suggestive of phagocytosis/wound debridement (movie 9, available at www.jneurosci.org as supplemental mate- 
A

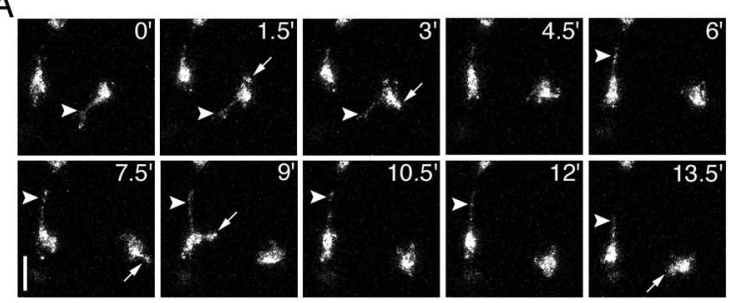

B

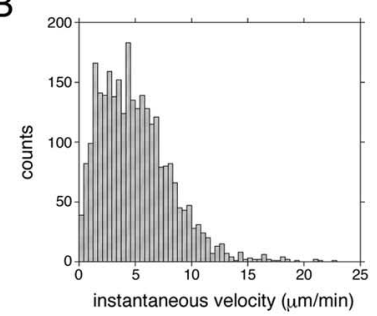

Figure 3. In situ motility of perilesional microglia. $\boldsymbol{A}$, Migratory microglia demonstrated transient forward protrusions (white arrows) and long-lasting trailing processes (white arrowheads). Scale bar, $30 \mu \mathrm{m}$. $\boldsymbol{B}$, Distribution of lateral velocities of continuously migrating microglia measured at 90 s intervals ( $n=2815$ measurements from 47 cells; 4 slices from 4 different mice). Each frame in $\boldsymbol{A}$ is a two-dimensional projection representing a stack of six images $8 \mu \mathrm{m}$ apart (40 $\mu \mathrm{m}$ depth).

prisingly, the average individual cell S was similar to the instantaneous population average at $4.98 \pm 0.25 \mu \mathrm{m} / \mathrm{min}$ (range, $1.90-11.42 \mu \mathrm{m} / \mathrm{min}$; median, $4.80 \mu \mathrm{m} /$ $\min )$. The average $P$ was determined to be $1.0 \pm 0.01 \mathrm{~min}$ (range, $0.75-1.55 \mathrm{~min}$; median, $0.98 \mathrm{~min})$. Path length $(\mathrm{S} \times \mathrm{P})$, which is the distance traveled before a change in direction, was $4.95 \pm 0.23 \mu \mathrm{m}$ (range, 1.73-10.42 $\mu \mathrm{m}$; median, $4.86 \mu \mathrm{m}$ ). Finally, a measure of the distribution of the cell population, or dispersion $\left(\mathrm{S}^{2} \times \mathrm{P}\right)$, was $27.02 \pm 2.88 \mu \mathrm{m}^{2} / \mathrm{min}$ (range, $3.41-$ $119.02 \mu \mathrm{m}^{2} / \mathrm{min}$; median, $23.07 \mu \mathrm{m}^{2}$ / $\mathrm{min})$. Thus, migratory perilesional microrial). These cells represented only $\sim 5-30 \%$ of the total number of cells in each field. We conclude that perilesional microglia are highly locomotory $1 \mathrm{~d}$ after injury, and many exhibit longdistance migration.

To examine lateral $(x-y)$ velocities of continuously migratory perilesional microglia (see Materials and Methods), we plotted instantaneous velocity versus time from individual cell data pooled from four independent 90 min experiments (Fig. 3B) (47 cells; $n=2815$ observations). As a population, microglia migrated an average of $5.02 \pm 0.06 \mu \mathrm{m} / \mathrm{min}(\sim 300 \mu \mathrm{m} / \mathrm{h})$ with peak bursts beyond $10 \mu \mathrm{m} / \mathrm{min}(\sim 600 \mu \mathrm{m} / \mathrm{h})$. Velocities fluctuated during migration. However, there was no dominant or consistent periodicity (data not shown). We conclude that activated perilesional microglia migrate at high, fluctuating velocities.

\section{Autonomous, nonbiased migration of microglia in situ}

In all experiments, the majority of locomotory cells appeared to migrate in a stochastic manner (Fig. $4 \mathrm{~A}$ ) (movie 11, available at www.jneurosci.org as supplemental material). Highly directed cells migrating linearly toward the lesion were rarely observed. To systematically determine whether microglia migrate collectively and directionally, we overlaid $y$-axis-normalized microglial cell tracks plotted from a common origin (Fig. $4 B$ ). Cells appeared to migrate as autonomous agents moving in all directions with no evidence for bulk flow. When the 47 trajectories are averaged, the resulting path is found in the positive $x$ direction (Fig. $4 B$, inset). However, the magnitude (net displacement) of this mean cell path was minimal $(17.72 \mu \mathrm{m})$. As expected, the $\mathrm{CI}$ for the population was only 0.09 (see Materials and Methods). We conclude that perilesional microglial migration is mostly independent and not highly directional.

\section{Perilesional microglial migration can be modeled as a random walk}

The autonomous and meandering trajectories of continuous perilesional microglial migrators is reminiscent of a random walk (Zygourakis, 1996). Indeed, the average ratio of net to total migration distance was just 0.25 . To determine whether the 47 longdistance migrating cells analyzed above could be modeled as random walkers, we determined the mean-squared displacement of the entire population ( $n=2815$ intervals; four independent experiments). We found that mean absolute displacement was proportional to the square root of time (Fig. $4 B$ ), consistent with a random walk process $\left(R^{2}=0.9998\right)$.

To further characterize the migration of perilesional microglia, we fitted the cell path analysis to the PRW model (Dunn, 1983; Othmer et al., 1988). All cell trajectories, individually $\left(R^{2}>\right.$ $0.90)$, reasonably fit the PRW model (data not shown). Not sur- glia display high velocities but are not highly directionally persistent.

\section{CCR5 modulates microglial migration}

CC chemokines acting at the CCR5 receptor, such as macrophage inflammatory protein- $1 \alpha / \mathrm{CC}$ chemokine ligand 3 (MIP- $1 \alpha /$ CCL3), MIP-1 $\beta / C C L 4$, and regulated on activation, normal T-cell expressed and secreted (RANTES)/CCL5, are upregulated in neurological diseases and in response to brain injury (Ghirnikar et al., 1996; Hausmann et al., 1998; Bajetto et al., 2002; Babcock et al., 2003; Marella and Chabry, 2004). These factors have been thought to mediate primarily leukocyte homing to brain lesions (Bajetto et al., 2002). Recently, CCR5 function has been shown to be important for chemotaxis of resident brain macrophages, the microglia, in vitro and has been thought to mediate similar functions in vivo (Bajetto et al., 2002; Marella and Chabry, 2004). However, this role must be reconciled with the predominantly autonomous, random walk migration of perilesional microglia that we observed. Miller et al. (2003) proposed that chemokines, in certain situations, modulate specific physical parameters of cellular migration such as speed or protrusive events. To test this hypothesis in perilesional microglia, we directly observed microglial locomotion $24 \mathrm{~h}$ after lesion under conditions of chemokine receptor CCR5 inhibition with the highly specific, small-molecule antagonist TAK-779 (see Materials and Methods). A dose-response study was performed, revealing that the $40 \mu \mathrm{m}$ dose resulted in a significant $43 \%$ reduction in the number of migratory cells per subfield reflected as percentage of migratory microglia $(p<0.05)$ (Fig. $5 A)$ (movie 12, middle, available at www.jneurosci.org as supplemental material). Although migration was inhibited, cell motility in general remained highly active. Many of these "arrested" cells under CCR5 blockade with $40 \mu \mathrm{M}$ TAK-779 displayed repeated protrusive lunges in various directions as if attempting to migrate; however, little net displacement was exhibited. There was no difference in the number of directional protrusive events (see Materials and Methods) between these migration-arrested microglia and normally migrating microglia during a 30 min observation period ( $p=0.67$; $t$ test; mean scores, $5.6 \pm 0.5$ and $5.4 \pm 0.4$, respectively), suggesting that directional control is independent of CCR5 activation. Microglial migration could be rescued to $86 \%$ of predrug conditions after $24 \mathrm{~h}$ of drug washout (Fig. 5A) (movie 12, right, available at www.jneurosci.org as supplemental material). The number of viable cells per field did not differ significantly between any of the three phases of recording, further ruling out a toxic effect of $40 \mu \mathrm{M}$ TAK-779 treatment (Fig. 5B) ( $p=0.89$; ANOVA). Thus, blockade of CCR5 significantly hinders locomotion of perilesional microglia but not directional protrusive events. 
A

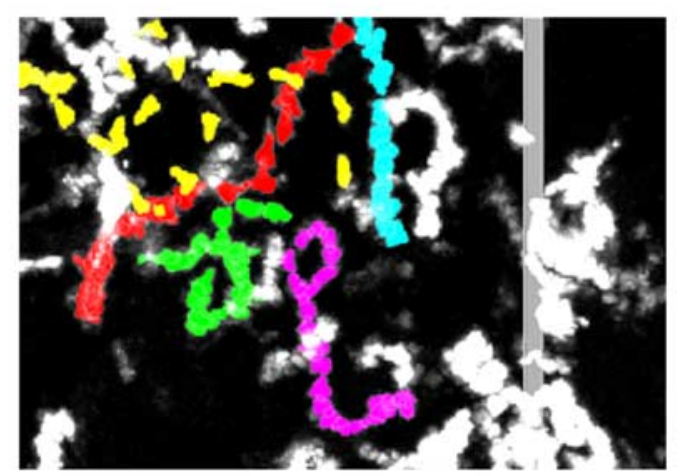

B

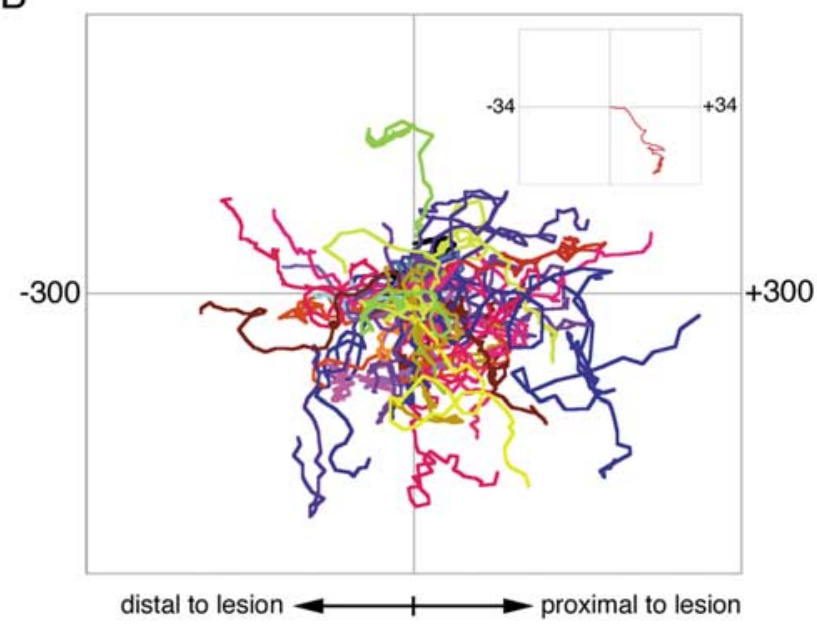

C

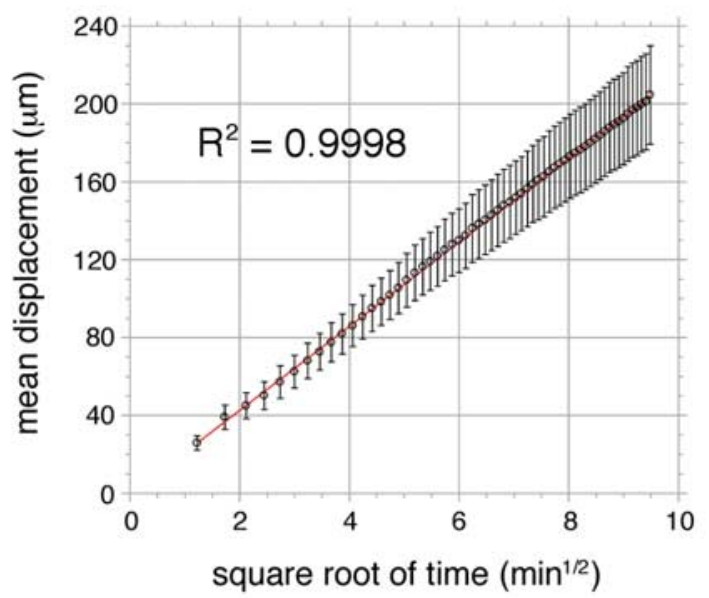

Figure 4. Random walk perilesional microglial migration. $\boldsymbol{A}$, Perilesional microglial migration is not collective nor highly directed (movie 11, available at www.jneurosci.org as supplemental material). $\boldsymbol{B}$, Normalized cell paths of continuously migrating microglia plotted from a common origin demonstrates migration of microglia in all directions with no obvious evidence for directional bulk flow ( $n=47$ cells; 4 slices from 4 different mice). The mean (inset) and median (data not shown) paths demonstrate only a minor bias toward the lesion. Units are in micrometers. $\boldsymbol{C}$, The mean absolute displacement of continuously migrating microglia plotted as a function of the square root of time. The close fit to a straight line on this transformed scale demonstrates that migration proceeds essentially as a random walk $\left(R^{2}=0.9998\right)$.

To determine the effects of CCR5 blockade on the enduring migratory microglia, we performed PRW analysis at the $40 \mu \mathrm{M}$ dose. The average cell speed decreased $27 \%$ compared with baseline conditions under CCR5 inhibition $(p<0.01)$ but
A

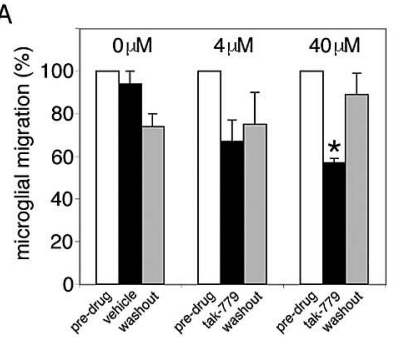

B

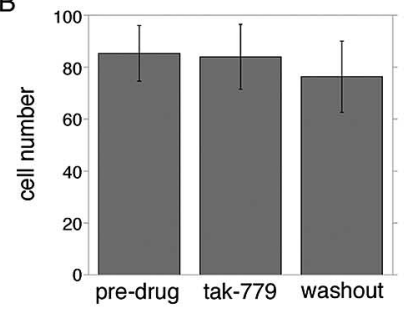

C

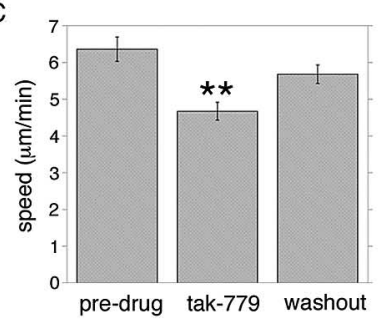

D

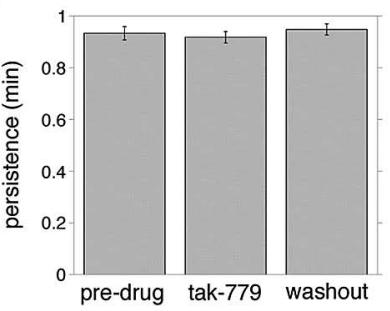

$\mathrm{E}$

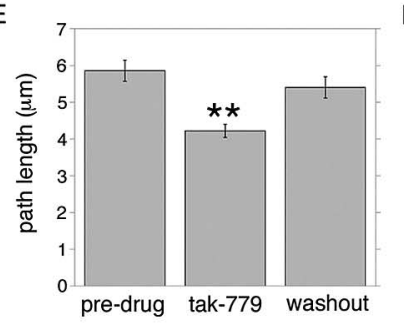

$\mathrm{F}$

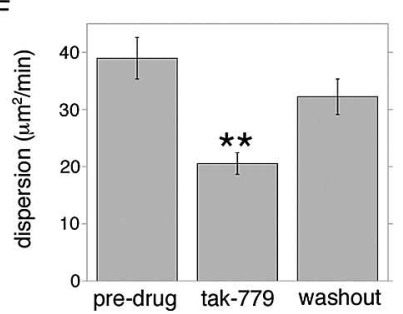

G

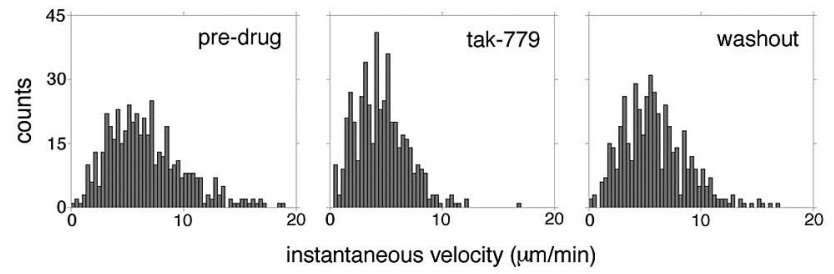

Figure 5. CCR5 modulates microglial migration. Individual slices were recorded sequentially in the absence of drug, in the presence of the highly selective CCR5 inhibitor TAK-779, and after $24 \mathrm{~h}$ of drug washout ( $n=3$ slices from 3 different mice; each dose). $A$, TAK-779 treatment at $40 \mu \mathrm{m}$ reversibly reduced the number of migratory microglial cells as reflected by percentage of migratory microglia $\left({ }^{*} p<0.05\right)$. B, Cell viability with $40 \mu \mathrm{m}$ TAK-779 did not differ between the three recording phases as demonstrated by cell number per recording field ( $p=0.89$ ). PRW analysis was applied to the $40 \mu \mathrm{m}$ TAK-779 experiments revealing significant $\left({ }^{* *} p<\right.$ 0.01) differences between predrug conditions and TAK-779 for cell speed (C), path length $(\boldsymbol{E})$, and dispersion $(\boldsymbol{F})$. $\boldsymbol{D}$, There were no differences in directional persistence times. All parameters could be rescued to near pretreatment values after drug washout. G, TAK-779 treatment (40 $\mu \mathrm{M}$ ) resulted in a leftward shift of the distribution histogram of instantaneous velocities (mean, $4.57 \pm 0.11 \mu \mathrm{m} / \mathrm{min}$ ) compared with pretreatment (mean, $6.65 \pm 0.15 \mu \mathrm{m} / \mathrm{min}$ ). Drug washout (mean, $6.30 \pm 0.15 \mu \mathrm{m} / \mathrm{min}$ ) rescued velocity distribution to near-baseline conditions.

could be rescued to $90 \%$ of baseline levels with washout (Fig. $5 C)$. Consistent with the no-drug effect on directional protrusive events noted above, directional persistence times were unaffected (Fig. 5D). Average path length and dispersion were decreased by $28 \%$ (Fig. $5 E)(p<0.01)$ and $47 \%$ (Fig. $5 F)(p<$ 0.01 ), respectively. Path length returned to $92 \%$ of baseline and dispersion to $83 \%$ after washout. Also supporting the PRW analysis, TAK-779 resulted in a leftward shift of the distribution histogram of instantaneous velocities, whereas washout resulted in a nearly identical distribution as before treatment (Fig. 5G). Thus, we conclude that CCR5 activation 
A

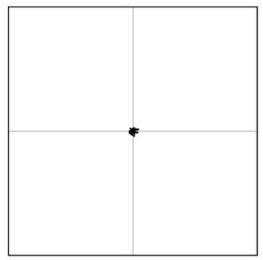

deafferentation

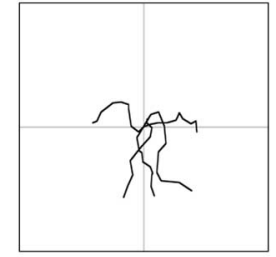

stab lesion

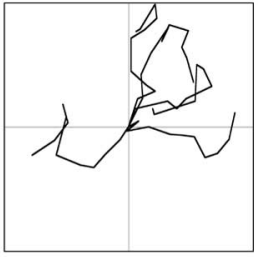

hiv-1 tat protein
B

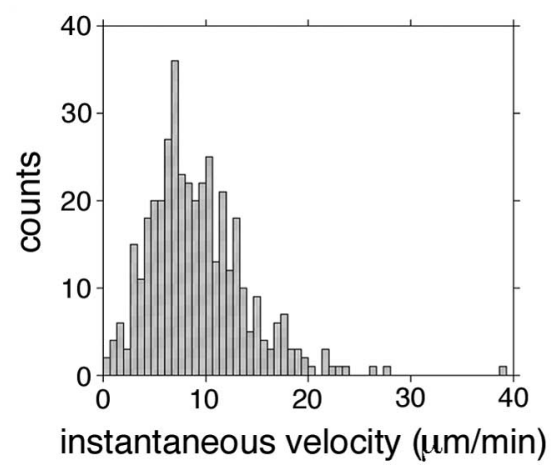

Figure 6. Microglial migration responses are stimulus dependent. $\boldsymbol{A}$, Trajectories of five representative migrating cells for each condition were traced for a 15 min recording period and plotted from a common origin. Microglia migrated extensively in the perilesional thalamus at $3 \mathrm{~d}$ (middle) but not in the contralateral hippocampus at the site of denervation at $3 \mathrm{~d}$ (left). Microglia activated by HIV-1 tat protein migrated the greatest distances. Plots measure, $150 \times$ $150 \mu \mathrm{m}$. B, Microglia activated by an intracerebroventricular injection of HIV-1 tat protein $24 \mathrm{~h}$ before slice collection migrated approximately twice as fast as cells in the perilesional thalamus at $24 \mathrm{~h}$ (compare with Fig. 3B) (see movie 14, available at www.jneurosci.org as supplemental material).

modulates speed, but not directional persistence, of migrating microglia.

\section{Induction and quality of microglial migration is stimulus dependent}

To examine dynamic behavior of activated microglia in indirectly damaged regions, we collected slices 3 and $7 \mathrm{~d}$ after FSL and rendered movies from the hippocampal stratum oriens contralateral ( $\mathrm{cSO})$ to the lesion. This region is deafferented by the wound (Hausmann et al., 1998; Carbonell et al., 2005) (W. S. Carbonell and J. W. Mandell, unpublished observations). In contrast to the widespread, continuous migration at the primary lesion at $24 \mathrm{~h}$ (Fig. $6 \mathrm{~A}$, middle), only a small population of cells $(<10 \%)$ migrated in the cSO and for brief bursts of only a few cell lengths (Fig. $6 \mathrm{~A}$, left). These microglia demonstrated highly dynamic processes (movie 13, available at www.jneurosci.org as supplemental material). At $7 \mathrm{~d}$ after injury, microglial activation in the $\mathrm{cSO}$ was diminished as demonstrated by very low to undetectable $\mathrm{IB}_{4}$ labeling of cells (data not shown). We conclude that activated microglia in the deafferented cSO, while possessing highly dynamic processes, do not appear to migrate extensively.

HIV-1 tat protein is a key regulator of viral replication (Rappaport et al., 1999). It has also been found to be neurotoxic in nanomolar quantities (Philippon et al., 1994) and induces migration of microglia in vitro (Lokensgaard et al., 2001). To test the hypothesis that HIV-1 tat protein would induce migration of microglia in situ, we prepared brain slices for time-lapse confocal microscopy $24 \mathrm{~h}$ after an intracerebroventricular injection of 2.0 $\mathrm{nM}$ recombinant tat. Tat-activated microglia in situ were highly migratory (Fig. 6A, right, $B$ ) (movie 14, left, available at www. jneurosci.org as supplemental material) with an average instantaneous velocity of $9.20 \pm 0.24 \mu \mathrm{m} / \mathrm{min}(\sim 550 \mu \mathrm{m} / \mathrm{h})$ and peak bursts beyond $16 \mu \mathrm{m} / \mathrm{min}(>1000 \mu \mathrm{m} / \mathrm{h})$. Migratory paths were meandering, similar to cells in the perilesional thalamus (Fig. $6 \mathrm{~A}$, right) (movie 14, left, available at www.jneurosci.org as supplemental material). Slices from control animals injected with vehicle alone (data not shown) or deactivated HIV-1 tat protein (movie 14, right, available at www.jneurosci.org as supplemental material) demonstrated only limited microglial activation and no migration. Thus, the induction and quality of microglial migration is not uniform but highly stimulus dependent.

\section{Discussion}

In neurological diseases and diverse brain insults, microglial cells incite and promote the innate neuroinflammatory response responsible for brain repair (Kreutzberg, 1996; Becher et al., 2000; Streit et al., 2004). However, this inflammatory cascade, like that of the peripheral tissues in the body, can become pathogenic and contribute to the progression of disease processes. Therefore, understanding microglial function is essential for designing rational approaches for therapeutic modulation of this response. In this study, we combined an in vivo focal lesion model with laserscanning confocal time-lapse microscopy to investigate the migratory behavior of activated microglia in acutely isolated living brain slices from adult mice. These results define dynamic parameters of the migratory response of activated perilesional microglia to focal brain injury in living tissue from adult mice for the first time.

Dailey and Waite (1999) demonstrated that the FITC-tagged microglial-specific lectin $\mathrm{IB}_{4}$ from Griffonia simplicifolia can be used to vitally label microglia in hippocampal slices from early postnatal rats. They exploited these properties to study microglial dynamics after tissue injury with confocal microscopy using the trauma of the slicing procedure as the activation stimulus. Their important investigations shed new light on the dynamics of the early microglial reaction (Stence et al., 2001; Grossmann et al., 2002). However, likely because of the acute nature of their activation stimulus (i.e., the brain slicing procedure), they observed only a small degree of cell migration (Stence et al., 2001). Indeed, Nimmerjahn et al. (2005) found no migration of microglia for up to $5.5 \mathrm{~h}$ after a laser lesion in mice in vivo. In contrast, our methods allow the neuroinflammatory response to develop unperturbed in vivo before slice collection and, therefore, are particularly suited for the study of mature reactive phenotypes such as long-distance microglial migration. These phenotypes are not displayed in brain slices from naive adult mice after $24 \mathrm{~h}$ of culture, demonstrating that the slice models are not equivalent (movie 8, available at www.jneurosci.org as supplemental material). These results validate the use of acutely isolated brain slices after in vivo lesion in adult mice for the dynamic study of mature microglial reactions.

Here, we focused our investigation on resident microglial migration. We selected the $1 \mathrm{~d}$ time point after stab lesion to perform discrete analysis of migratory parameters. Although greater numbers of migratory cells were observed at the $3 \mathrm{~d}$ time point, the excessive numbers of overlapping and interacting cells hindered discrete two-dimensional analysis. We were also concerned about a larger contribution of blood macrophages to the $\mathrm{IB}_{4^{-}}$ positive cell population because of the nature of our injury model (Peters et al., 1991). Indeed, it has been classically thought that the number of macrophages of vascular origin in brain injury models with blood-brain barrier disruption was extensive. How- 
ever, recent studies have demonstrated that resident microglia considerably outnumber invading macrophages at all time points after various injuries (Fujita et al., 1998; Babcock et al., 2003; Schilling et al., 2003; Vallières and Sawchenko, 2003). For instance, Fujita et al. (1998) estimated $102 \pm 10$ macrophages per cubic millimeter of tissue $24 \mathrm{~h}$ after stab lesion based on immunohistochemical and morphological criteria. Using this estimate, we would expect less than one cell per $350 \times 350 \times 40 \mu \mathrm{m}$ field to be of blood origin in our analyzed movies at $1 \mathrm{~d}$. Therefore, the great majority of migratory cells observed at $1 \mathrm{~d}$ after stab lesion are likely resident microglia.

Our finding of autonomous, random walk locomotion in the perilesional thalamus is not consistent with the classical chemotaxic gradient hypothesis of microglial migration after injury. The suggestion that chemokines may exert effects via modulation of motile behavior of individual cells rather than promotion of directional bulk flow of cell populations seems particularly salient in light of these data (Miller et al., 2003; Wei et al., 2003). A prominent chemokine receptor implicated in resident microglial migration in the literature is CCR5. It has been shown to be constitutively expressed in resting microglia (Simpson et al., 2000; Otto et al., 2001) and upregulated in diverse pathological states (Mennicken et al., 2002; Glabinski et al., 2003; Natale et al., 2003). Additionally, many CCR 5 ligands such as MIP- $1 \alpha /$ CCL3, MIP-1 $\beta / C C L 4$, and RANTES/CCL5 are released after neural injury (Ghirnikar et al., 1996; Babcock et al., 2003; Marella and Chabry, 2004). Consistent with this idea, we found that in situ inhibition of the CC chemokine receptor CCR5 with the potent and highly specific small-molecule inhibitor TAK-779 (Baba et al., 1999) could reduce the extent of perilesional microglial migration by $>40 \%$ and decrease migration speed of enduring migratory cells by nearly $30 \%$. In contrast, directional persistence was not affected. CCR5 is also known to be variably expressed in astroglia in normal and pathological states (Mennicken et al., 2002; Lee et al., 2005). However, the acute effects of TAK-779 on microglial migration as well as the rescue experiments with drug washout strongly support a microglial receptor-based effect rather than an indirect effect through inhibition of astrocytic CCR5 or effects at the level of transcription. Therefore, we conclude that activation of CCR5 is an important regulator of biophysical parameters of microglial migration.

The incomplete inhibition of microglial migration with TAK779 , which we observed in the present study, is consistent with the findings of Marella and Chabry (2004) in an in vitro prion model. Together, our results suggest that other chemokine and nonchemokine receptors (Nolte et al., 1997; Walter et al., 2003) likely contribute to microglial migration after brain insult. Other possible chemokine receptor candidates include CCR1, CCR3, CX3CR1, and CXCR3 (Maciejewski-Lenoir et al., 1999; Bajetto et al., 2002; Flynn et al., 2003); however, relative contributions likely depend on injury stimulus. The intrinsic chemokines for CCR5 have been studied previously after stab lesion (Ghirnikar et al., 1996) and in other injury models (Baker et al., 1999; Otto et al., 2001; Babcock et al., 2003; Marella and Chabry, 2004). A candidate common to all models is RANTES/CCL5. However, Marella and Chabry (2004) demonstrated that maximal inhibition of microglial migration in vitro in response to prion with anti-RANTES/CCL5 antibodies was only $\sim 30 \%$ (compared with $\sim 50 \%$ with $40 \mu \mathrm{M}$ TAK-779), supporting a contribution of other CCR5 ligands to microglial migration. This topic deserves additional investigation.

The role of microglial migration in the neuroinflammatory response has not been studied extensively but is thought to be related to cytokine receptor profiles as well as release and expression of ligands (Bajetto et al., 2002; Flynn et al., 2003). These parameters, in turn, highly depend on the injury stimulus and extent of damage. Indeed, microglial responses are thought to manifest in a graded stereotypic pattern (Kreutzberg, 1996). Thus, the degree of microglial migration in tissue may represent a barometer of neuropathologic severity. However, the dynamic responses of microglia in the face of varying injury types has not been studied previously. In contrast to the robust locomotory response in the perilesional thalamus, we found that microglia in the contralateral denervated stratum oriens of the hippocampus were primarily nonmigratory, multipolar cells with highly active cellular processes. This may be similar to the graded response of microglia in the lethal and sublethal facial nerve lesion models (Streit and Kreutzberg, 1988). Additionally, microglia activated by an intracerebroventricular injection of $\mathrm{HIV}-1$ tat protein migrated nearly twice as fast as those in the perilesional thalamus. This may be explained by both the direct neurotoxic effects of lentiviral tat (Philippon et al., 1994) and the known mimicry of CC chemokines by tat (Albini et al., 1998). HIV-1 tat effects on CC chemokine and chemokine receptor expression, including CCR5 and its ligands (Weiss et al., 1999; McManus et al., 2000), are also noted. Indeed, these data indirectly support our findings (see above) that CCR5 is involved in modulation of microglial migration velocity. Thus, our results are consistent with the idea of graded, context-dependent microglial activation and regulation.

In summary, the in situ confocal time-lapse approach used in the current study revealed the remarkable extent and diversity of the microglial migratory response to focal brain injury. These results put forth a new model of microglial migration after neural injury, whereby a stochastic, rather than directed, migration strategy is used to patrol certain areas of damage. This is similar to what has been proposed for lymphocyte trafficking and detection of antigen in lymph nodes (Wei et al., 2003). This strategy is advantageous over linear trajectories, because it allows a wider and more uniform coverage of tissue (Fig. $2 B$, middle), assuring proper allocation of resources to all areas in need of attention, not just the primary lesion. However, this does not exclude directed microglial migration in other injury contexts (McGladeMcCulloh et al., 1989; Carbonell et al., 2005) or during development. The role of chemokines in certain situations may be to enhance the probability of microglia locating damage by modulating physical parameters of migration such as velocity. Finally, recruitment and quality of microglial migration depends on injury context. These results further our understanding of the complex microglial response to neural injury and have important implications for the development of treatment strategies for modulating neuroinflammation in human disease and injury.

\section{References}

Albini A, Ferrini S, Benelli R, Sforzini S, Giunciuglio D, Aluigi MG, Proudfoot AE, Alouani S, Wells TN, Mariani G, Rabin RL, Farber JM, Noonan DM (1998) HIV-1 tat protein mimicry of chemokines. Proc Natl Acad Sci USA 95:13153-13158.

Baba M, Nishimura O, Kanzaki N, Okamoto M, Sawada H, Iizawa Y, Shiraishi M, Aramaki Y, Okonogi K, Ogawa Y, Meguro K, Fujino M (1999) A small-molecule, nonpeptide CCR5 antagonist with highly potent and selective anti-HIV-1 activity. Proc Natl Acad Sci USA 96:5698-5703.

Babcock AA, Kuziel WA, Rivest S, Owens T (2003) Chemokine expression by glial cells directs leukocytes to sites of axonal injury in the CNS. J Neurosci 23:7922-7930.

Bajetto A, Bonavia R, Barbero S, Schettini G (2002) Characterization of chemokines and their receptors in the central nervous system: physiopathological implications. J Neurochem 82:1311-1329. 
Baker CA, Lu ZY, Zaitsev I, Manuelidis L (1999) Microglial activation varies in different models of Creutzfeldt-Jakob disease. J Virol 73:5089-5097.

Becher B, Prat A, Antel JP (2000) Brain-immune connection: immunoregulatory properties of CNS-resident cells. Glia 29:293-304.

Benveniste EN (1997) Role of macrophages/microglia in multiple sclerosis and experimental allergic encephalomyelitis. J Mol Med 75:165-173.

Carbonell WS, Mandell JW (2003) Transient neuronal but persistent astroglial activation of ERK/MAP kinase after focal brain injury in mice. J Neurotrauma 20:327-336.

Carbonell WS, Murase S, Horwitz AF, Mandell JW (2005) Infiltrative microgliosis: activation and long-distance migration of subependymal microglia following periventricular insults. J Neuroinflammation 2:5.

Dailey ME, Waite M (1999) Confocal imaging of microglial cell dynamics in hippocampal slice cultures. Methods 18:222-230.

Duan Y, Haugabook SJ, Sahley CL, Muller KJ (2003) Methylene blue blocks cGMP production and disrupts directed migration of microglia to nerve lesions in the leech CNS. J Neurobiol 57:183-192.

Dunn GA (1983) Characterizing a kinesis response: time averaged measures of cell speed and directional persistence. Agents Actions [Suppl] $12: 14-33$.

Fanales-Belasio E, Moretti S, Nappi F, Barillari G, Micheletti F, Cafaro A, Ensoli B (2002) Native HIV-1 tat protein targets monocyte-derived dendritic cells and enhances their maturation, function, and antigenspecific T cell responses. J Immunol 168:197-206.

Flynn G, Maru S, Loughlin J, Romero IA, Male D (2003) Regulation of chemokine receptor expression in human microglia and astrocytes. J Neuroimmunol 136:84-93.

Fujita T, Yoshimine T, Maruno M, Hayakawa T (1998) Cellular dynamics of macrophages and microglial cells in reaction to stab wounds in rat cerebral cortex. Acta Neurochir (Wien) 140:275-279.

Ghirnikar RS, Lee YL, He TR, Eng LF (1996) Chemokine expression in rat stab wound brain injury. J Neurosci Res 46:727-733.

Glabinski AR, Bielecki B, Kolodziejski P, Han Y, Selmaj K, Ransohoff RM (2003) TNF-alpha microinjection upregulates chemokines and chemokine receptors in the central nervous system without inducing leukocyte infiltration. J Interferon Cytokine Res 23:457-466.

Grossmann R, Stence N, Carr J, Fuller L, Waite M, Dailey ME (2002) Juxtavascular microglia migrate along brain microvessels following activation during early postnatal development. Glia 37:229-240.

Hausmann EHS, Berman NEJ, Wang Y-Y, Meara JB, Wood GW, Klein RM (1998) Selective chemokine mRNA expression following brain injury. Brain Res 788:49-59.

Knight B, Laukaitis C, Akhtar N, Hotchin NA, Edlund M, Horwitz AF (2000) Visualizing muscle cell migration in situ. Curr Biol 10:576-585.

Kobori N, Clifton GL, Dash P (2002) Altered expression of novel genes in the cerebral cortex following experimental brain injury. Brain Res Mol Brain Res 104:148-158.

Kreutzberg GW (1996) Microglia: a sensor for pathological events in the CNS. Trends Neurosci 19:312-318.

Lee H, Jun Y, Choi J, Kim J, Carp RI, Kim Y (2005) The expression of RANTES and chemokine receptors in the brains of scrapie-infected mice. J Neuroimmunol 158:26-33.

Lokensgaard JR, Hu S, Hegg CC, Thayer SA, Gekker G, Peterson PK (2001) Diazepam inhibits HIV-1 tat-induced migration of human microglia. J Neurovirol 7:481-486.

Maciejewski-Lenoir D, Chen S, Feng L, Maki R, Bacon KB (1999) Characterization of fractalkine in rat brain cells: migratory and activation signals for CX3CR1-expressing microglia. J Immunol 163:1628-1635.

Maheshwari G, Lauffenburger DA (1998) Deconstructing (and reconstructing) cell migration. Microsc Res Tech 43:358-368.

Marella M, Chabry J (2004) Neurons and astrocytes respond to prion infection by inducing microglia recruitment. J Neurosci 24:620-627.

McGlade-McCulloh E, Morrissey AM, Norona F, Muller KJ (1989) Individual microglia move rapidly and directly to nerve lesions in the leech central nervous system. Proc Natl Acad Sci USA 86:1093-1097.

McManus CM, Weidenheim K, Woodman SE, Nunez J, Hesselgesser J, Nath A, Berman JW (2000) Chemokine and chemokine-receptor expression in human glial elements: induction by the HIV protein, tat, and chemokine autoregulation. Am J Pathol 156:1441-1453.

Mennicken F, Chabot JG, Quirion R (2002) Systemic administration of kainic acid in adult rat stimulates expression of the chemokine receptor CCR5 in the forebrain. Glia 37:124-138.

Miller MJ, Wei SH, Cahalan MD, Parker I (2003) Autonomous T cell trafficking examined in vivo with intravital two-photon microscopy. Proc Natl Acad Sci USA 100:2604-2609.

Murase S-I, Horwitz AF (2002) Deleted in colorectal carcinoma and differentially expressed integrins mediate the directional migration of neural precursors in the rostral migratory stream. J Neurosci 22:3568-3579.

Natale JE, Ahmed F, Cernak I, Stoica B, Faden AI (2003) Gene expression profile changes are commonly modulated across models and species after traumatic brain injury. J Neurotrauma 20:907-927.

Nelson PT, Soma LA, Lavi E (2002) Microglia in diseases of the central nervous system. Ann Med 34:491-500.

Nimmerjahn A, Kirchhoff F, Helmchen F (2005) Resting microglial cells are highly dynamic surveillants of brain parenchyma in vivo. Science 305:1314-1318.

Nolte C, Kirchhoff F, Kettenmann H (1997) Epidermal growth factor is a motility factor for microglial cells in vitro: evidence for EGF receptor expression. Eur J Neurosci 9:1690-1698.

Othmer HG, Dunbar SR, Alt W (1988) Models of dispersal in biological systems. J Math Biol 26:263-298.

Otto VI, Stahel PF, Rancan M, Kariya K, Shohami E, Yatsiv I, Eugster HP, Kossmann T, Trentz O, Morganti-Kossmann MC (2001) Regulation of chemokines and chemokine receptors after experimental closed head injury. NeuroReport 12:2059-2064.

Peters A, Palay SL, Webster H deF (1991) The neuroglial cells. In: The fine structure of the nervous system, Ed 3, pp 304-311. New York: Oxford UP.

Petersen MA, Dailey ME (2004) Diverse microglial motility behaviors during clearance of dead cells in hippocampal slices. Glia 46:195-206.

Philippon V, Vellutini C, Gambarelli D, Harkiss G, Arbuthnott G, Metzger D, Roubin R, Filippi P (1994) The basic domain of the lentiviral tat protein is responsible for damages in mouse brain: involvement of cytokines. Virology 20:519-529.

Rappaport J, Joseph J, Croul S, Alexander G, Del Valle L, Amini S, Khalili K (1999) Molecular pathway involved in HIV-1-induced CNS pathology: role of viral regulatory protein, tat. J Leuk Bio 65:458-465.

Schilling M, Besselmann M, Leonhard C, Mueller M, Ringelstein EB, Kiefer R (2003) Microglial activation precedes and predominates over macrophage infiltration in transient focal cerebral ischemia: a study in green fluorescent protein transgenic bone marrow chimeric mice. Exp Neurol 183:25-33.

Simpson J, Rezaie P, Newcombe J, Cuzner ML, Male D, Woodroofe MN (2000) Expression of the beta-chemokine receptors CCR2, CCR3 and CCR5 in multiple sclerosis central nervous system tissue. J Neuroimmunol 108:198-200.

Stence N, Waite M, Dailey ME (2001) Dynamics of microglial activation: a confocal time-lapse analysis in hippocampal slices. Glia 33:256-266.

Streit WJ, Kreutzberg GW (1988) Response of endogenous glial cells to motor neuron degeneration induced by toxic ricin. J Comp Neurol 268:248-263.

Streit WJ, Mrak RE, Griffin WST (2004) Microglia and neuroinflammation: a pathological perspective. J Neuroinflammation 1:14.

Vallières L, Sawchenko PE (2003) Bone marrow-derived cells that populate the adult mouse brain preserve their hematopoietic identity. J Neurosci 23:5197-5207.

Walter L, Franklin A, Witting A, Wade C, Xie Y, Kunos G, Mackie K, Stella N (2003) Nonpsychotropic cannabinoid receptors regulate microglial cell migration. J Neurosci 23:1398-1405.

Ware MF, Wells A, Lauffenburger DA (1998) Epidermal growth factor alters fibroblast migration speed and directional persistence reciprocally and in a matrix-dependent manner. J Cell Sci 111:2423-2432.

Wei SH, Parker I, Miller MJ, Cahalan MD (2003) A stochastic view of lymphocyte motility and trafficking within the lymph node. Immunological Rev 195:136-159.

Weiss JM, Nath A, Major EO, Berman JW (1999) HIV-1 tat induces monocyte chemoattractant protein-1-mediated monocyte transmigration across a model of the human blood-brain barrier and upregulates CCR5 expression on human monocytes. J Immunol 163:2953-2959.

Zygourakis K (1996) Quantification and regulation of cell migration. Tissue Eng 2:1-26. 